\title{
Natural anticoagulants limit lipopolysaccharide-induced pulmonary coagulation but not inflammation
}

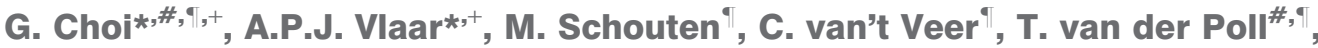 \\ M. Levi ${ }^{\#}$ and M.J. Schultz ${ }^{\star,+}$
}

ABSTRACT: Pulmonary coagulopathy and hyperinflammation may contribute to an adverse outcome in sepsis. The present study determines the effects of natural inhibitors of coagulation on bronchoalveolar haemostasis and inflammation in a rat model of endotoxaemia.

Male Sprague-Dawley rats were randomised to treatment with normal saline, recombinant human activated protein C (APC), plasma-derived antithrombin (AT), recombinant human tissue factor pathway inhibitor (TFPI), heparin or recombinant tissue plasminogen activator (tPA). Rats were intravenously injected with lipopolysaccharide (LPS), which induced a systemic inflammatory response and pulmonary inflammation. Blood and bronchoalveolar lavage were obtained at 4 and $16 \mathrm{~h}$ after LPS injection, and markers of coagulation and inflammation were measured.

LPS injection caused an increase in the levels of thrombin-AT complexes, whereas plasminogen activator activity was attenuated, both systemically and within the bronchoalveolar compartment. Administration of APC, AT and TFPI significantly limited LPS-induced generation of thrombin-AT complexes in the lungs, and tPA stimulated pulmonary fibrinolytic activity. However, none of the agents had significant effects on the production of pulmonary cytokines, chemokines, neutrophil influx and myeloperoxidase activity.

Natural inhibitors of coagulation prevent bronchoalveolar activation of coagulation, but do not induce major alterations of the pulmonary inflammatory response in rat endotoxaemia.

KEYWORDS: Acute lung injury, coagulation, fibrinolysis, protein C, sepsis

evere sepsis is a clinical syndrome characterised by a systemic inflammatory response and activation of coagulation, potentially leading to intravascular depositions of fibrin and microvascular thrombosis, or bleeding related to uncontrolled consumption of coagulation factors [1]. Under physiological circumstances, the activation of coagulation is regulated by natural inhibitors of coagulation, i.e. activated protein $\mathrm{C}$ (APC), antithrombin (AT) and tissue factor pathway inhibitor (TFPI). In sepsis, these anticoagulant systems are impaired, most likely due to massive consumption and downregulation by inflammatory mediators, which form the rationale for therapeutic restoration of these natural anticoagulant pathways [2]. Unfortunately, both plasma-derived AT [3] and recombinant human (rh)TFPI [4] have failed to reduce patient mortality in severe sepsis. The pivotal phase III clinical trial with rhAPC showed a significant increase in patient survival [5], but there is ongoing debate about the exact mechanisms by which APC prevents death.

One of the proposed mechanisms by which APC exerts its protective effects relates to pathways involving the lungs. The lungs are the most frequently involved organ system in sepsisrelated multiple organ failure. Notably, APC treatment causes more rapid resolution of respiratory failure during sepsis [6] and limits both coagulation and influx of neutrophils in the pulmonary compartment in experimental lung injury $[7,8]$. In a relatively limited number of patients with sepsis, lung-protective effects have also been suggested during treatment with AT [9] and TFPI [10], but these were not confirmed in larger phase III clinical trials [3, 4]. In the current study, using a rat model of endotoxaemia, it was hypothesised that APC, AT and TFPI would have differential effects on pulmonary coagulation and inflammation, potentially explaining different outcomes in human sepsis.
AFFILIATIONS

Depts of *Intensive Care Medicine,

\#Internal Medicine,

- Centre for Experimental and

Molecular Medicine, Centre for

Infection and Immunity, and

+Laboratory of Experimental Intensive Care and Anaesthesiology, Academic Medical Centre, University of Amsterdam, Amsterdam, The Netherlands.

CORRESPONDENCE

G. Choi

Dept of Intensive Care Medicine

Academic Medical Centre

University of Amsterdam

C3-423

Meibergdreef 9

1105 AZ Amsterdam

The Netherlands

Fax: 31206972988

E-mail: godachoi@mail.com

Received:

December 202006

Accepted after revision:

May 142007

\section{SUPPORT STATEMENT}

The present study was supported by the Netherlands Organization for Health Research and Development (ZonMW; Den Haag, the

Netherlands). M.J. Schultz was the recipient of a ZonMW-VENI grant 2004 (project number 016.056.001)

STATEMENT OF INTEREST

None declared.

European Respiratory Journal Print ISSN 0903-1936 Online ISSN 1399-3003 


\section{MATERIAL AND METHODS}

\section{Rats}

Male Sprague-Dawley rats (200-250 g) were purchased from Harlan (The Hague, the Netherlands). The rats were allowed to acclimatise to laboratory conditions for $\geqslant 7$ days $(12 / 12 \mathrm{~h}$ daynight cycle at $22^{\circ} \mathrm{C}$ ). The Institutional Animal Care and Use Committee of the Academic Medical Centre (University of Amsterdam, Amsterdam, the Netherlands) approved all experiments. All animals were handled in accordance with the guidelines prescribed by the Dutch legislation and the international guidelines on protection, care and handling of laboratory animals.

\section{Study design}

Endotoxaemia was induced by administration of $7.5 \mathrm{mg} \cdot \mathrm{kg}^{-1}$ lipopolysaccharide (LPS) from Escherichia coli 0111:B4 (Sigma, St. Louis, MO, USA) into the penile vein under isoflurane (3\%) anaesthesia. Pilot studies with this model demonstrated that LPS injection led to a transient hyperacute systemic inflammatory response, with systemic tumour necrosis factor (TNF)- $\alpha$, interleukin (IL)-6 and cytokine-induced neutrophil chemoattractant (CINC)-3 levels peaking within 1-2 h. Thereafter, these pro-inflammatory mediators were undetectable in the circulation, with prolonged effects on systemic and pulmonary coagulopathy $\leqslant 24 \mathrm{~h}$. No major histopathological changes in lung tissue were observed, other than neutrophil infiltration at $16 \mathrm{~h}$ after LPS injection. Rats were randomised to placebo (normal saline) or treatment with one of the natural anticoagulants: APC, AT or TFPI ( $n=8$ per group). Additionally, heparin and tissue plasminogen activator (tPA) were involved in the randomisation process. Four healthy rats without endotoxaemia were used as controls. All agents were administered in bolus injections of $2 \mathrm{~mL} \cdot \mathrm{kg}^{-1}, 30 \mathrm{~min}$ before injection of LPS. Therapeutic doses were determined using data from previous studies [4, 10-14]. All the agents and doses that were used are described in table 1 . Considering the plasma clearance, rats sacrificed at $16 \mathrm{~h}$ after LPS injection received additional injections of APC, TFPI, heparin or tPA at 6 and $12 \mathrm{~h}$. All rats were administered the same volume of fluid $\left(2 \mathrm{~mL} \cdot \mathrm{kg}^{-1}\right)$ at 6 and $12 \mathrm{~h}$.

Rats were sacrificed with intraperitoneal injections of ketamine $80 \mathrm{mg} \cdot \mathrm{kg}^{-1}$ (Eurovet, Bladel, the Netherlands) and medetomidine $0.5 \mathrm{mg} \cdot \mathrm{kg}^{-1}$ (Novartis, Arnhem, the Netherlands) at 4 and $16 \mathrm{~h}$ after LPS injection. Blood was collected from the inferior vena cava in citrated $(0.109 \mathrm{M})$ vacutainer tubes. The right lung was ligated and the left lung was lavaged three times with $2 \mathrm{~mL}$ ice-cold normal saline. Right lungs were weighed and homogenised in four volumes (i.e. four times lung weight (in $\mathrm{mg}$ ), in $\mu \mathrm{L}$ ) of sterile saline using a tissue homogeniser (Biospec Products, Bartlesville, OK, USA). Total cell numbers in each lavage sample were determined by an automated cell counter (Coulter Counter; Coulter Electronics, Hialeah, FL, USA). Neutrophil counts in lavage fluids were performed on Giemsa-stained cytospin preparations.

For coagulation assays, plasma and cell-free supernatants from bronchoalveolar lavage were used. For cytokine and chemokine measurements in lungs, supernatants were used from lung homogenates which were diluted 1:1 in lysis buffer $\left(150 \mathrm{nmol} \cdot \mathrm{L}^{-1} \mathrm{NaCl} ; 15 \mathrm{mmol} \cdot \mathrm{L}^{-1}\right.$ Tris; $1 \mathrm{mmol} \cdot \mathrm{L}^{-1} \mathrm{MgCl}_{2}-\mathrm{H}_{2} \mathrm{O}$; $1 \mathrm{mmol} \cdot \mathrm{L}^{-1} \mathrm{CaCl}_{2} ; 1 \%$ Triton $\mathrm{X}-100$; and $100 \mu \mathrm{g} \cdot \mathrm{mL}^{-1}$ pepstatin A, leupeptin and aprotinin).

\section{Assays}

Thrombin-antithrombin complexes (TATc; Behring, Marburg, Germany) and fibrin degradation products (FDP; Asserachrom D-Di; Diagnostica Stago, Asnières-sur-Seine, France) were measured using ELISA. AT, plasminogen activator activity (PAA) and plasminogen activator inhibitor (PAI)-1 activity were measured by automated amidolytic assays [15-17]. Levels of TNF- $\alpha$, IL- 6 and CINC-3 were measured using ELISA (R\&D Systems, Abingdon, UK). Myeloperoxidase (MPO) activity was determined by measuring the $\mathrm{H}_{2} \mathrm{O}_{2}$ dependent oxidation of 3,3',5,5'-tetramethylbenzidine, and was expressed as activity per gram of lung tissue [18].

\section{Statistical analysis}

All data are expressed as mean \pm SEM or median (interquartile range), as appropriate. Comparisons between the experimental groups and the saline-treated placebo group were performed using one-way ANOVA or the Kruskal-Wallis test, followed by post hoc Dunnett's or Dunn's tests, depending on data distribution. A p-value $<0.05$ was considered statistically significant.

\section{RESULTS \\ Coagulation and fibrinolysis}

Compared with controls which were not injected with LPS, endotoxaemia caused increased generation of TATc (fig. 1), an effect which was attenuated by APC, AT and TFPI at both 4 and $16 \mathrm{~h}$ after LPS injection (fig. 1a). Plasma PAA was significantly decreased after LPS injection; tPA increased PAA to levels exceeding assay maximum at both time-points $(\mathrm{p}<0.001$ versus saline; fig. $1 \mathrm{~b})$. With AT treatment, plasma PAA was also significantly higher than with saline at both time-points $(p<0.01$ versus saline; fig. $1 b)$.

\section{TABLE 1 Pharmaceutical agents and doses used}

\begin{tabular}{|c|c|c|c|c|c|}
\hline Biological equivalent & Abbreviation & Agent & Manufacturer & Dose & [Ref.] \\
\hline Activated protein C & APC & Drotrecogin alpha (activated) & Eli Lilly (Indianapolis, IN, USA) & $500 \mu \mathrm{g} \cdot \mathrm{g}^{-1}$ & {$[12]$} \\
\hline Antithrombin III & AT & Plasma-derived antithrombin III & Baxter (Vienna, Austria) & $250 \mathrm{U} \cdot \mathrm{kg}^{-1}$ & {$[12,13]$} \\
\hline Tissue factor pathway inhibitor & TFPI & Tifacogin & Chiron (Emeryville, CA, USA) & $1.5 \mathrm{mg} \cdot \mathrm{kg}^{-1}$ & {$[4,10,11]$} \\
\hline Heparan sulfate & Heparin & Unfractionated heparin & Leo Pharma (Ballerup, Denmark) & $300 \mathrm{U} \cdot \mathrm{kg}^{-1}$ & [12] \\
\hline Tissue-type plaminogen activator & tPA & Tenecteplase & $\begin{array}{l}\text { Boehringer Ingelheim (Ingelheim, } \\
\text { Germany) }\end{array}$ & $1.25 \mathrm{mg} \cdot \mathrm{kg}^{-1}$ & {$[14]$} \\
\hline
\end{tabular}



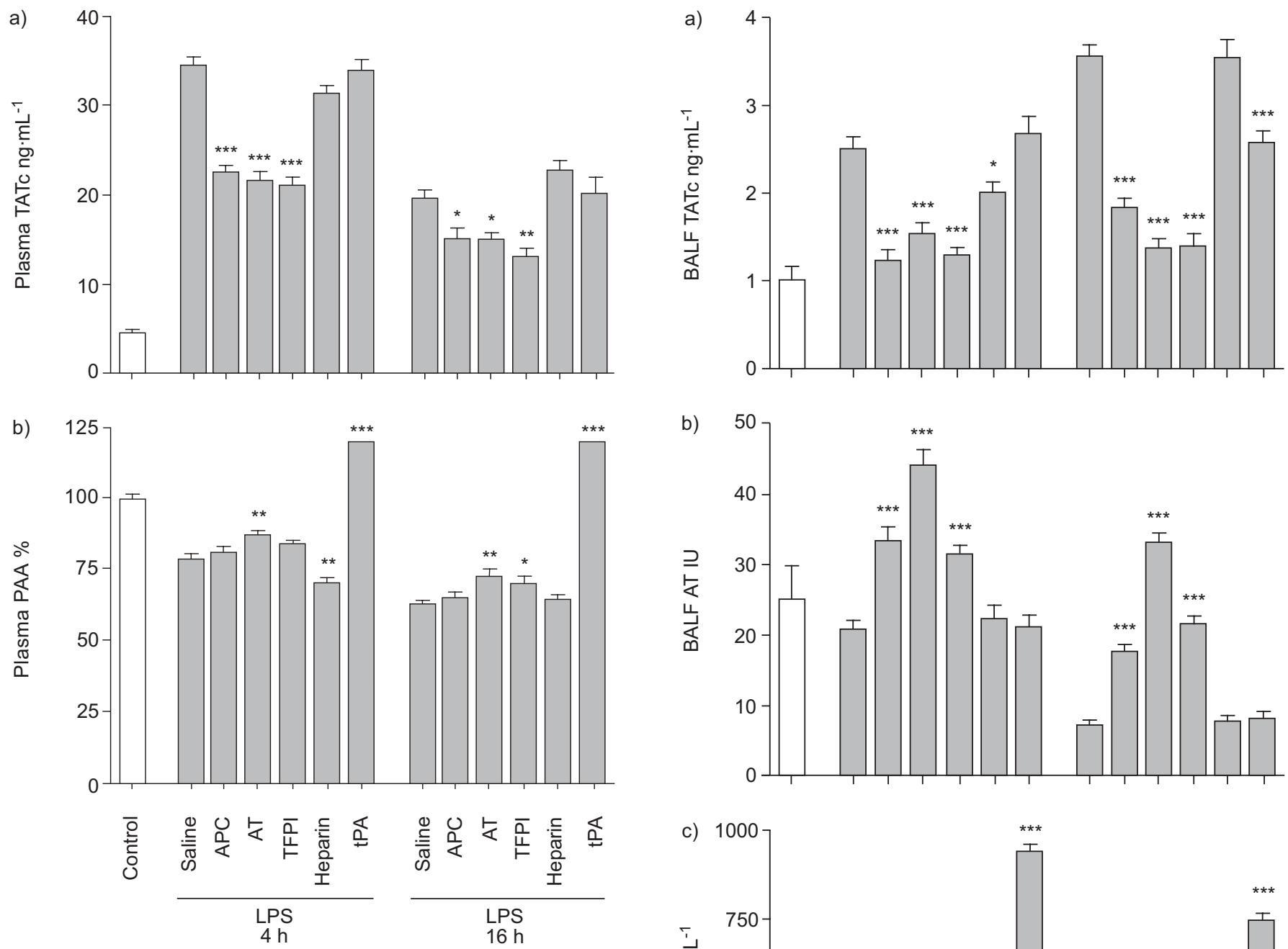

FIGURE 1. The effects of anticoagulants 4 and $16 \mathrm{~h}$ after injection of lipopolysaccharide (LPS; Escherichia coli O111:B4) on plasma levels of a) thrombin-antithrombin complexes (TATc) and b) systemic plasminogen activator activity (PAA). Control, $n=4$; saline, $n=11$ in the LPS $4 \mathrm{~h}$ group and $n=12$ in the LPS $16 \mathrm{~h}$ group; recombinant human activated protein $C$ (APC), $n=8$; plasma-derived human antithrombin (AT), $n=8$; recombinant human tissue factor pathway inhibitor (TFPI), $n=8$; heparin, $n=8$; tissue-type plasminogen activator (tPA), $n=8$. Error bars represent SEM. The $p$-values were calculated according to Dunnett's test. *: $p<0.05$ versus saline; ${ }^{* *}: p<0.01$ versus saline; ${ }^{* \star *}: p<0.001$ versus saline.

Furthermore, bronchoalveolar levels of TATc were increased by endotoxaemia (fig. 2). APC, AT and TFPI limited LPSinduced generation of bronchoalveolar TATc $(\mathrm{p}<0.001$ versus saline for both time-points; fig. 2a), thereby preventing a decrease in AT activity and limiting FDP generation (figs $2 b$ and c). Heparin treated rats also showed less bronchoalveolar TATc but only at $4 \mathrm{~h}$ (fig. 2a), while AT activity was not different from saline treated rats (fig. 2b). Pulmonary PAA in lungs was significantly reduced by endotoxaemia, with concurrently enhanced PAI-1 activity in lungs (fig. 3). tPA increased bronchoalveolar PAA and FDP levels (both $p<0.001$ versus saline for both time-points; figs $3 \mathrm{a}$ and $2 \mathrm{c}$ ), while abolishing enhanced PAI-1 activity $(\mathrm{p}<0.001$ versus saline for both time-points; fig. 3b).

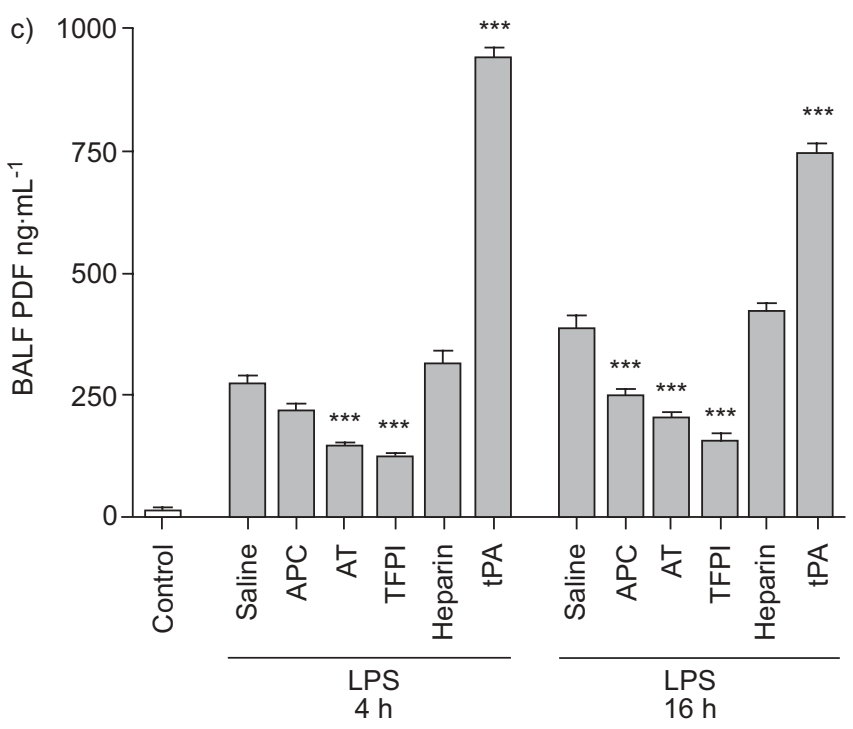

FIGURE 2. The effects of anticoagulants 4 and $16 \mathrm{~h}$ after injection of lipopolysaccharide (LPS; Escherichia coli O111:B4) on levels of a) thrombinantithrombin complexes (TATc), b) antithrombin activity (AT) and c) fibrin degradation products (FDP) in bronchoalveolar lavage fluid (BALF). Control, $n=4$; saline, $n=11$ in the LPS $4 \mathrm{~h}$ group and $n=12$ in the LPS $16 \mathrm{~h}$ group; recombinant human activated protein $\mathrm{C}$ (APC), $\mathrm{n}=8$; plasma-derived human antithrombin (AT), $n=8$; recombinant human tissue factor pathway inhibitor (TFPI), $n=8$; heparin, $n=8$; tissue-type plasminogen activator (tPA), $n=8$. Error bars represent SEM. The $p$-values were calculated according to Dunnett's test. ${ }^{*}: p<0.05$ versus saline; $\star * *: p<0.001$ versus saline. 

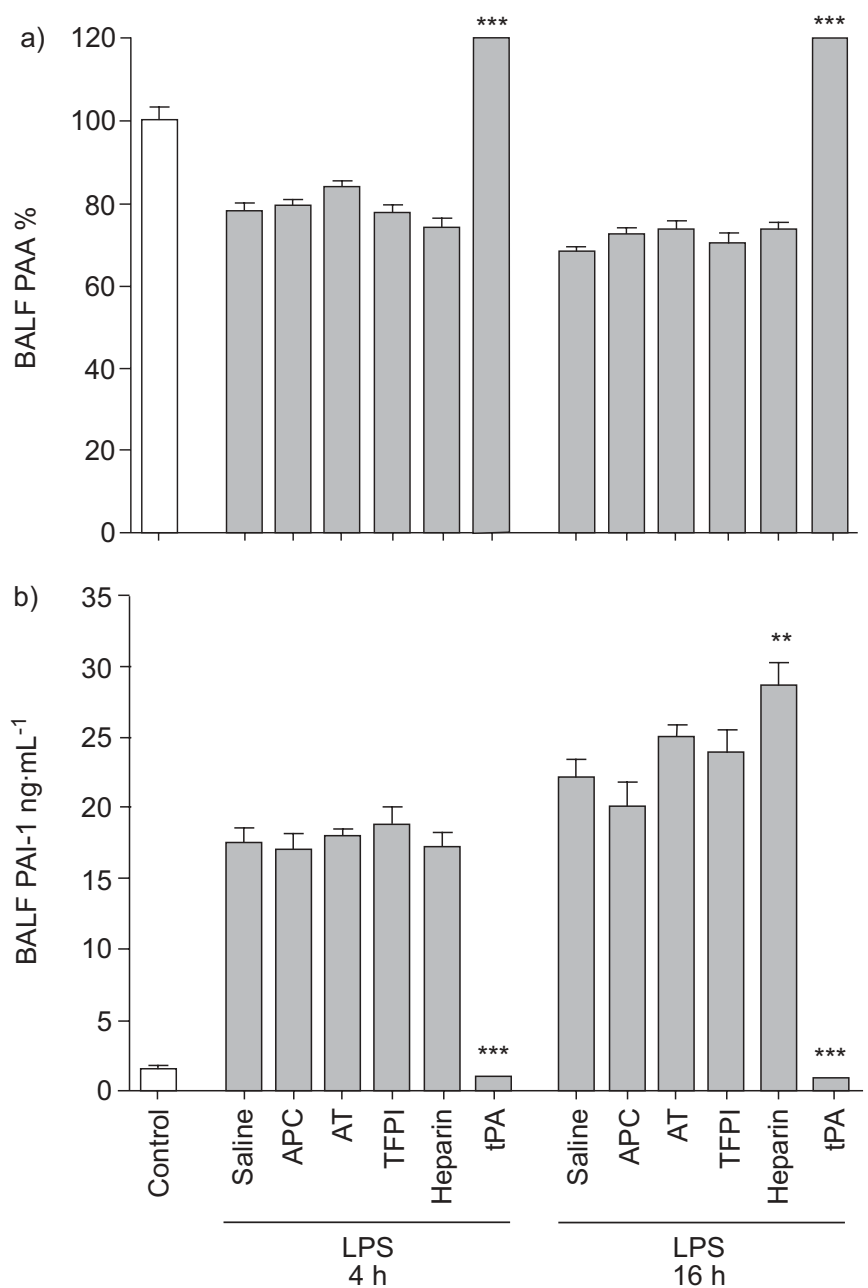

FIGURE 3. The effects of anticoagulants 4 and $16 \mathrm{~h}$ after injection of lipopolysaccharide (LPS; Escherichia coli O111:B4) on a) plasminogen activator activity (PAA) and b) levels of plasminogen activator inhibitor type 1 (PAl-1) in bronchoalveolar lavage fluid (BALF). Control, $n=4$; saline, $n=11$ in the LPS $4 \mathrm{~h}$ group and $n=12$ in the LPS $16 \mathrm{~h}$ group; recombinant human activated protein $\mathrm{C}$ (APC), $n=8$; plasma-derived human antithrombin (AT), $n=8$; recombinant human tissue factor pathway inhibitor (TFPI), $n=8$; heparin, $n=8$; tissue-type plasminogen activator (tPA), $n=8$. Error bars represent SEM. The $p$-values were calculated according to Dunnett's test. ${ }^{* *}: p<0.01$ versus saline; ${ }^{* \star *}$ : $p<0.001$ versus saline.

\section{Inflammatory response}

During rat endotoxaemia, TFPI treatment enhanced IL-6 generation in lungs at $16 \mathrm{~h}(\mathrm{p}<0.01$ versus saline; fig. $4 \mathrm{~b})$; all other agents did not induce significant changes in pulmonary levels of TNF- $\alpha$, IL- 6 and CINC-3. Inflammatory cell influx into the lungs was not observed at $4 \mathrm{~h}$ after LPS injection. There was an increase in neutrophil counts in the lungs during endotoxaemia at $16 \mathrm{~h}$, but there were no differences in the number of neutrophils between the anticoagulant groups and the saline group (table 2). Finally, MPO in lung tissue was not influenced by any treatment (table 2).

\section{DISCUSSION}

The present authors have shown that the application of systemic anticoagulant agents alters bronchoalveolar coagulation
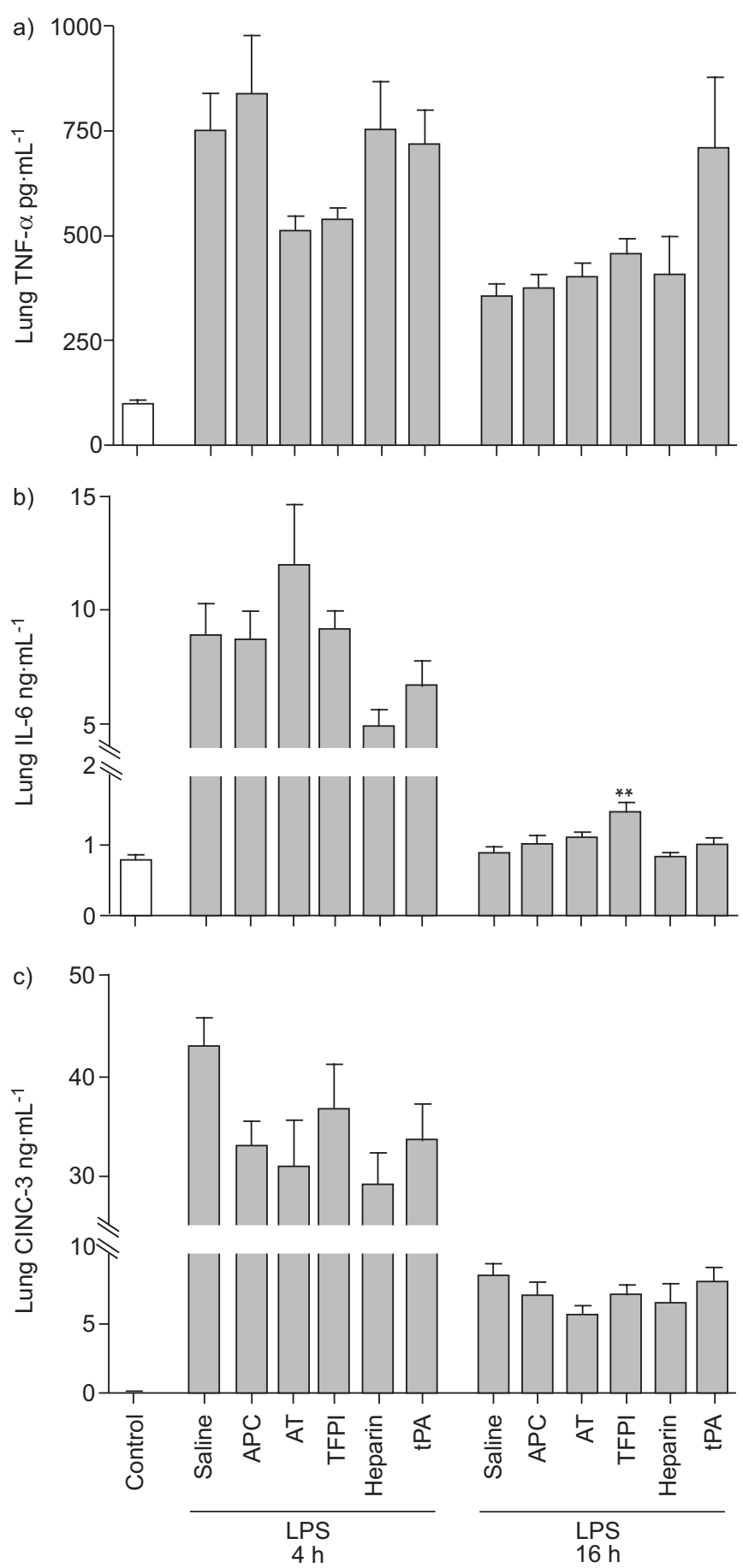

FIGURE 4. a) Tumour necrosis factor (TNF)- $\alpha$, b) interleukin (IL)-6 and c) cytokine-induced neutrophil chemoattractant (CINC)-3, determined in lung homogenates 4 and $16 \mathrm{~h}$ after injection of lipopolysaccharide (LPS; Escherichia coli O111:B4). Control, $n=4$; saline, $n=11$ in the LPS 4 h group and $n=12$ in the LPS $16 \mathrm{~h}$ group; recombinant human activated protein $\mathrm{C}$ (APC), $n=8$; plasma-derived human antithrombin (AT), $n=8$; recombinant human tissue factor pathway inhibitor (TFPI), $\mathrm{n}=8$; heparin, $\mathrm{n}=8$; tissue-type plasminogen activator (tPA), $\mathrm{n}=8$. Error bars represent SEM. The $p$-values were calculated according to Dunn's test. **: $p<0.01$ versus saline

during endotoxaemia. APC, AT and TFPI had major effects on limiting thrombin generation, both systemically and in the bronchoalveolar compartment. However, the various agents 


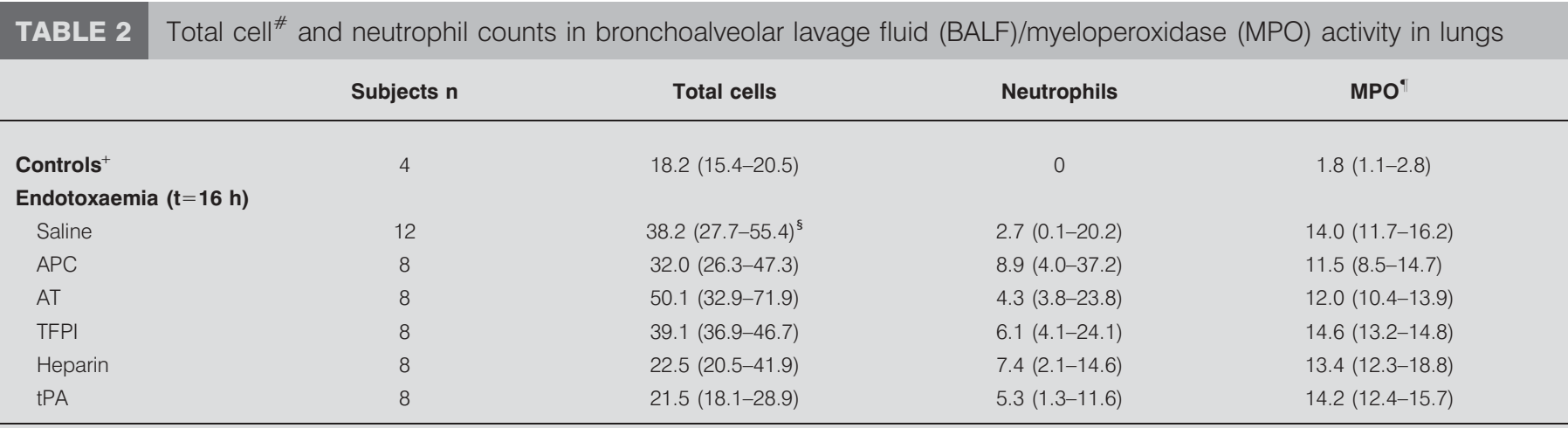

Data are expressed as median (interquartile range) $\times 10^{4}$ per $\mathrm{mL}$ of BALF. APC: recombinant human activated protein C; AT: plasma-derived antithrombin; TFPI: recombinant human tissue factor pathway inhibitor; tPA: recombinant tissue-type plasminogen activator. ${ }^{*}$ : cell counts were performed $16 \mathrm{~h}$ after injection with lipopolysaccharide (Escherischia coli O111:B4); ${ }^{\bullet}$ : measured in units per lung weight (in g); ${ }^{+}$: controls are uninfected rats; ${ }^{\varsigma}$ : $p=0.008$ and $p>0.05$ (all versus saline) according to Kruskal-Wallis and Dunn's post-tests, respectively.

had very limited effects on pulmonary inflammation, suggesting that the inflammatory response is not significantly directed by changes in pulmonary coagulation itself, at least not in the present rat model of endotoxaemia. The question "what mechanisms account for differences in clinical outcome with various anticoagulant strategies?" remains unresolved.

In the pathogenesis of endotoxin-induced lung injury, an important role has been attributed to neutrophils. UCHIBA et al. [19] demonstrated that endotoxin-induced pulmonary vascular injury in Wistar rats is mainly mediated by activated neutrophils. Using this model, it was shown that APC [20], AT with or without heparin co-administration [21], and TFPI [15] had inhibitory effects on activated neutrophils, thereby limiting neutrophil accumulation, MPO activity and cytokine generation in the lungs in the first hour after endotoxin administration. Also, it was shown that neutrophil influx is significantly inhibited by rhAPC infusion in healthy volunteers challenged with intrapulmonary endotoxin [7]. In the present rat model, neutrophil accumulation and MPO activity in the lungs were not significantly altered by any of the agents administered. This discrepancy with previous studies may have been caused by differences in endotoxin delivery, species, investigated time-points and the subsequent different roles for neutrophil involvement in the experimental models. It is noteworthy that in most previous studies, inflammatory effects were investigated in the hyperacute phase after endotoxin challenge (e.g. $1 \mathrm{~h})$. The present results illustrate that it is relevant to include later time-points in which the local host response may significantly be altered, although systemic effects have subsided.

Heparin is a broadly used anticoagulant that acts by binding AT and facilitating its anticoagulant activity. Heparin had been shown to exert lung protective effects during endotoxaemia in sheep [16], while low molecular weight heparin had been able to limit lung injury in endotoxaemic pigs [17] and mice [18]. In the present experiment, heparin had a mild anticoagulant effect and a modest antifibrinolytic effect: pulmonary TATC and systemic PAA were decreased at $4 \mathrm{~h}$ after LPS injection, while pulmonary PAI-1 activity was increased after $16 \mathrm{~h}$. This is in contrast with in vitro data suggesting that heparins have pro-fibrinolytic effects [22]. The limited anticoagulant effects of heparin may have been caused by inadequate dosing, but it is also possible that increased AT consumption had led to impaired anticoagulant activity. Notably, treatment with heparin did not result in altered inflammatory response within the lungs. Opposing the anticoagulant strategies, tPA was used to stimulate fibrinolysis. Increased fibrinolytic activity did not affect neutrophil influx into the lungs, nor did it alter generation of inflammatory mediators.

A most important limitation of the study is the rat model of endotoxaemia. Lung injury models with either direct endotracheal instillation of endotoxin or via endotoxaemia have routinely been used in experimental studies due to its relative ease and good reproducibility. It should be noted that endotoxin-induced lung injury is a simplified model of patients with pneumonia or sepsis with acute lung injury. If acute lung injury is related to infectious processes, interference with coagulation could theoretically limit containment of the primary infection, promoting microbial dissemination [23]. Furthermore, anticoagulants were delivered by bolus injections, instead of continuous intravenous infusion. It may well be that bolus injections of anticoagulants are able to limit bronchoalveolar coagulation; however, for anti-inflammatory effects (e.g. neutrophil migration) [7] continuous infusion is needed to maintain constant plasma levels. Despite the limitations of the present model, it should be noted that the current results are in line with data from a study in patients after abdominal surgery for secondary peritonitis [24]. In that study, patients demonstrated a short-lived systemic inflammatory response syndrome after the surgical procedure, which was followed by a profound pro-coagulant response in the lungs [24]. The present model mimics this clinical pattern, at least in part: rat endotoxaemia also caused a transient systemic inflammatory response, while inducing pulmonary coagulopathy at later time-points. This finding is of interest, especially in the context of multiple injurious processes that patients may encounter during critical illness.

In conclusion, it has been demonstrated that systemic application of natural anticoagulants significantly inhibits bronchoalveolar activation of coagulation in a rat model of 
endotoxaemia, but does not lead to distinct effects on cytokine and chemokine production or neutrophil migration and activity. It remains uncertain whether controlled pulmonary coagulation significantly contributes to patient outcome and thus needs to be established in appropriate clinical trials.

\section{REFERENCES}

1 Levi M, Ten Cate H. Disseminated intravascular coagulation. N Engl J Med 1999; 341: 586-592.

2 Esmon CT. Role of coagulation inhibitors in inflammation. Thromb Haemost 2001; 86: 51-56.

3 Warren BL, Eid A, Singer P, et al. Caring for the critically ill patient. High-dose antithrombin III in severe sepsis: a randomized controlled trial. JAMA 2001; 286: 1869-1878.

4 Abraham E, Reinhart K, Opal S, et al. Efficacy and safety of tifacogin (recombinant tissue factor pathway inhibitor) in severe sepsis: a randomized controlled trial. JAMA 2003; 290: 238-247.

5 Bernard GR, Vincent JL, Laterre PF, et al. Efficacy and safety of recombinant human activated protein $\mathrm{C}$ for severe sepsis. N Engl J Med 2001; 344: 699-709.

6 Vincent JL, Angus DC, Artigas A, et al. Effects of drotrecogin alfa (activated) on organ dysfunction in the PROWESS trial. Crit Care Med 2003; 31: 834-840.

7 Nick JA, Coldren CD, Geraci MW, et al. Recombinant human activated protein $C$ reduces human endotoxininduced pulmonary inflammation via inhibition of neutrophil chemotaxis. Blood 2004; 104: 3878-3885.

8 van der Poll T, Levi M, Nick JA, Abraham E. Activated protein $\mathrm{C}$ inhibits local coagulation after intrapulmonary delivery of endotoxin in humans. Am J Respir Crit Care Med 2005; 171: 1125-1128.

9 Eisele B, Lamy M, Thijs LG, et al. Antithrombin III in patients with severe sepsis. A randomized, placebo-controlled, double-blind multicenter trial plus a meta-analysis on all randomized, placebo-controlled, double-blind trials with antithrombin III in severe sepsis. Intensive Care Med 1998; 24: 663-672.

10 Abraham E, Reinhart K, Svoboda P, et al. Assessment of the safety of recombinant tissue factor pathway inhibitor in patients with severe sepsis: a multicenter, randomized, placebo-controlled, single-blind, dose escalation study. Crit Care Med 2001; 29: 2081-2089.

11 Enkhbaatar P, Okajima K, Uchiba M, Isobe H, Okabe H. Recombinant tissue factor pathway inhibitor prevents lipopolysaccharide-induced systemic hypotension in rats by inhibiting excessive production of nitric oxide. Thromb Haemost 2001; 86: 1573-1577.

12 Schoots IG, Levi M, van Vliet AK, Maas AM, Roossink EH, van Gulik TM. Inhibition of coagulation and inflammation by activated protein $C$ or antithrombin reduces intestinal ischemia/reperfusion injury in rats. Crit Care Med 2004; 32: 1375-1383.

13 Hoffmann JN, Vollmar B, Laschke MW, et al. Adverse effect of heparin on antithrombin action during endotoxemia: microhemodynamic and cellular mechanisms. Thromb Haemost 2002; 88: 242-252.

14 Schoots IG, Levi M, van Vliet AK, Declerck PJ, Maas AM, van Gulik TM. Enhancement of endogenous fibrinolysis does not reduce local fibrin deposition, but modulates inflammation upon intestinal ischemia and reperfusion. Thromb Haemost 2004; 91: 497-505.

15 Enkhbaatar P, Okajima K, Murakami K, et al. Recombinant tissue factor pathway inhibitor reduces lipopolysaccharideinduced pulmonary vascular injury by inhibiting leukocyte activation. Am J Respir Crit Care Med 2000; 162: 1752-1759.

16 Schiffer ER, Reber G, de Moerloose P, Morel DR. Evaluation of unfractionated heparin and recombinant hirudin on survival in a sustained ovine endotoxin shock model. Crit Care Med 2002; 30: 2689-2699.

17 Darien BJ, Fareed J, Centgraf KS, et al. Low molecular weight heparin prevents the pulmonary hemodynamic and pathomorphologic effects of endotoxin in a porcine acute lung injury model. Shock 1998; 9: 274-281.

18 Slofstra SH, van 't Veer C, Buurman WA, Reitsma PH, Ten Cate H, Spek CA. Low molecular weight heparin attenuates multiple organ failure in a murine model of disseminated intravascular coagulation. Crit Care Med 2005; 33: 1365-1370.

19 Uchiba M, Okajima K, Murakami K, Okabe H, Takatsuki K. Endotoxin-induced pulmonary vascular injury is mainly mediated by activated neutrophils in rats. Thromb Res 1995; 78: 117-125.

20 Murakami K, Okajima K, Uchiba M, et al. Activated protein C prevents LPS-induced pulmonary vascular injury by inhibiting cytokine production. Am J Physiol 1997; 272: L197-L202.

21 Uchiba M, Okajima K, Murakami K. Effects of various doses of antithrombin III on endotoxin-induced endothelial cell injury and coagulation abnormalities in rats. Thromb Res 1998; 89: 233-241.

22 Lisman T, Adelmeijer J, Nieuwenhuis HK, de Groot PG. Enhancement of fibrinolytic potential in vitro by anticoagulant drugs targeting activated factor $X$, but not by those inhibiting thrombin or tissue factor. Blood Coagul Fibrinolysis 2003; 14: 557-562.

23 Schultz MJ, Haitsma JJ, Zhang H, Slutsky AS. Pulmonary coagulopathy as a new target in therapeutic studies of acute lung injury or pneumonia--a review. Crit Care Med 2006; 34: 871-877.

24 van Till JW, Levi M, Bresser P, Schultz MJ, Gouma DJ, Boermeester MA. Early procoagulant shift in the bronchoalveolar compartment of patients with secondary peritonitis. J Infect Dis 2006; 194: 1331-1339. 